\title{
Replication Competent Retrovirus
}

National Cancer Institute

\section{Source}

National Cancer Institute. Replication Competent Retrovirus. NCI Thesaurus. Code

C112045.

A retroviral vector that contains all the genes that are required for virion synthesis and is able to propagate after the host is infected. 